\title{
BMJ Open Quality Reducing acute kidney injury incidence and progression in a large teaching hospital
}

To cite: Sykes L, Sinha S, Hegarty J, et al. Reducing acute kidney injury incidence and progression in a large teaching hospital. BMJ Open Quality 2018;7:e000308. doi:10.1136/ bmjoq-2017-000308

Received 23 December 2017 Revised 13 September 2018 Accepted 12 October 2018

Check for updates

(c) Author(s) (or their employer(s)) 2018. Re-use permitted under CC BY-NC. No commercial re-use. See rights and permissions. Published by BMJ.

${ }^{1}$ Emergency Admission Unit, Salford Royal NHS Foundation Trust, Salford, UK

${ }^{2}$ Renal Department, Salford Royal NHS Foundation Trust, Salford, UK

${ }^{3}$ Information Management and Technology, Salford Royal NHS Foundation Trust, Salford, UK

${ }^{4}$ Quality Improvement, Salford Royal NHS Foundation Trust, Salford, UK

${ }^{5}$ Intensive Care, Salford Royal NHS Foundation Trust, Salford, UK

${ }^{6}$ Pharmacy, Salford Royal NHS Foundation Trust, Salford, UK ${ }^{7}$ Emergency Admissions Unit, Royal Preston Hospital, Preston, UK

Correspondence to Dr Lynne Sykes;

lynne.sykes@nhs.net

\section{ABSTRACT}

Acute kidney injury (AKI) is a common syndrome that is associated with significant mortality and cost. The Quality Improvement AKI Collaborative at Salford Royal Foundation Trust was established to review and improve both the recognition and management of AKI. This was a whole-system intervention to tackle AKI implemented as an alternative to employing separate AKI nurses. Our aims were to reduce the overall incidence of AKI by $10 \%$, to reduce hospital-acquired AKI by $25 \%$ and to reduce the progression of AKI from stage 1 to stage 2 or 3 by $50 \%$.

From 2014 to 2016, several multifaceted changes were introduced. These included system changes, such as inserting an e-alert for AKI into the electronic patient record, an online educational package and face-to-face teaching for AKI, and AKI addition to daily safety huddles. On 10 Collaborative wards, development of an AKI care bundle via multidisciplinary team (MDT) plan, do, study, act testing occurred.

Results showed a $15.6 \%$ reduction in hospitalwide-acquired $\mathrm{AKI}$, with a $22.3 \%$ reduction on the collaborative wards. Trust-wide rates of progression of AKI 1 to AKI 2 or 3 showed normal variation, whereas there was a $48.5 \%$ reduction in AKI progression on the Collaborative wards. This implies that e-alerts were ineffective in isolation. The Collaborative wards' results were a product of the educational support, bundle and heightened awareness of AKI.

A number of acute hospitals have demonstrated impactful successes in AKI reduction centred on a dedicated AKI nurse model plus e-alerting with supporting changes. This project adds value by highlighting another approach that does not require a new post with attendant rolling costs and risks. We believe that our approach increased our efficacy in acute care in our front-line teams by concentrating on embedding improved recognition and actions across the MDT.

\section{PROBLEM}

Acute kidney injury (AKI) is a common and serious syndrome affecting patients both in hospital and in the community. ${ }^{1}$ It is associated with significant morbidity and mortality. Recognition and management of AKI in the UK has been described as poor over the last decade by the national confidential enquiry into patient outcomes and death report 'Adding insult to injury'. 2

This study was designed to evaluate methods of reducing the incidence and progression of AKI through multifaceted interventions and to evaluate the impact of these on AKI incidence, AKI progression, in-hospital length of stay and mortality.

We used the Institute for Healthcare Improvement's Breakthrough Series Collaborative Model methodology. ${ }^{3}$ The AKI collaborative agreed three main aims:

- Reduce the overall incidence of AKI by $10 \%$.

- Reduce the incidence of hospital acquired AKI by $25 \%$.

- Reduce the progression of AKI from stage 1 to AKI stage 2 or 3 by $50 \%$.

Salford Royal NHS Foundation Trust (SRFT) serves a population of 230000 people and has a capacity of $>800$ inpatient beds. The local population has a high proportion of permanently sick or disabled patients $(6.7 \%)$ compared with the national average $(4 \%)$. Average life expectancy for Salford residents is 3 years less than for England as a whole, according to Public Health England 'Salford Health Profile 2015'.

SRFT is the lead Major Trauma Centre across Greater Manchester, the Comprehensive Stroke Centre and a tertiary referral centre for neurosurgery and renal services. It is also a national centre for intestinal failure and for metabolic diseases. The Trust is one of the most digitally mature Trusts in the National Health Service (NHS) and, as such, the electronic patient record (EPR) system lends itself to large, anonymous data collection and analysis.

SRFT centres around quality as its operating principle with a strategy supported by a quality improvement (QI) department since 2007 delivering change through 
collaboratives, microsystems, clinical quality academies, lean and flow interventions.

\section{BACKGROUND}

AKI has a reported incidence of 12\%-17.7\% from recent UK studies ${ }^{56}$ and it affects a wide range of patients both in hospital and the community. It occurs across a wide range of specialties, with most episodes of AKI occurring and being managed independent of nephrologists. ${ }^{7} \mathrm{AKI}$ is associated with longer lengths of hospital inpatient stay and has been associated with over 40000 deaths per annum in the UK. ${ }^{5}$ The cost of treating AKI is estimated at $£ 1$ billion per year, which is $1 \%$ of the annual NHS budget. ${ }^{5}$

$\mathrm{AKI}$ is associated with poorer long-term renal outcomes for patients. A large cohort study from Sweden showed higher incidence of chronic kidney disease at 1 year in patients who have had an AKI compared with patients without ( $6 \%$ vs $0.44 \%$ ), and the incidence of end-stage renal disease at 5 years was also higher $(3.9 \%$ vs $0.3 \%){ }^{8}$ Recovery after AKI varies significantly, but over $40 \%$ $(n=46)$ of de novo AKI failed to recover back to baseline in patients without pre-existing renal impairment in a small, single-centre study. $^{9}$

AKI came to the forefront of the UK national agenda following the national confidential enquiry into patient outcomes and death report of 2009 'Adding insult to injury'. This landmark enquiry highlighted global failings in recognition and management of AKI and showed that only $50 \%$ of care was considered good. ${ }^{2}$

There have been two recent QI studies, by Central Manchester NHS Foundation Trust (now part of Manchester University NHS Foundation Trust) and by Liverpool hospitals, that have attempted to tackle this problem. Each has employed the use of AKI nurses, AKI education, AKI bundles and AKI e-alerts. ${ }^{1011}$

Central Manchester NHS Foundation Trust employed two AKI nurses to screen their highly sensitive AKI e-alerts and to ensure their 10-point AKI priority care checklist was being completed. Alongside previous didactic teaching sessions, they developed novel opportunistic teaching with a four slide microteaching package. This generated a $28 \%$ reduction in AKI incidence, a $23 \%$ reduction in AKI-related length of stay and a trend towards improved mortality. ${ }^{10}$

The Liverpool hospitals took a similar approach, consisting of education, e-alert, bundle and a dedicated outreach team. This generated a $23.2 \%$ reduction in in-hospital mortality, a $25.9 \%$ reduction in 30-day mortality and a 2.6-day improvement in length of stay. ${ }^{11}$

The AKI Steering group and senior sponsors recognised the majority of AKI occurs outside of the renal ward. ${ }^{7}$ AKI can be seen as an 'illness barometer' that reflects the underlying severity of illness of the patient. Therefore, this project purposefully did not appoint AKI nurses and instead aimed to change Trust-wide culture through interventions that share recognition of, responsibility for, and management of, AKI.

\section{MEASUREMENT}

\section{Methods}

The NHS England national detection algorithm ${ }^{12}$ for AKI was programmed into our Telepath pathology system. Consequently, AKI results are automatically calculated in Telepath from available creatinine history. This in turn generates an electronic AKI flag that appears in the demographic banner of the EPR if sufficient deterioration in renal function is seen, according to the Kidney Disease, Improving Global Outcomes (KDIGO) definition of AKI. KDIGO define AKI stages as follows: stage 1 is an increase of more than $0.3 \mathrm{mg} / \mathrm{dL}(\geq 26.4 \mu \mathrm{mol} / \mathrm{L})$ or increase of 1.5 -fold to 2-fold from baseline, stage 2 is an increase 2-fold to 3-fold from baseline and stage 3 is an increase 3-fold or serum creatinine of $\geq 4.0 \mathrm{mg} / \mathrm{dL}(>354 \mu \mathrm{mol} / \mathrm{L})$ or initiation of renal replacement therapy. ${ }^{13}$

All pathology results are available in a structured query language database. The Information Management and Technology team was able to write a report to identify the data items needed for the indicators for the project. These data were pulled into Qlikview (Qlik, Pennsylvania, USA), a reporting tool that the group and the QI team can access. In Qlikview, all the incident data about each AKI alert are provided. These data include the date of occurrence for each incident and ward on which the incident occurred. The number of patients with an AKI stage of either stage 1, 2 or 3 was recorded.

AKI alerts within 48 hours of hospital admission were deemed to be community-acquired as, within this time frame, it is likely that the insult that caused the AKI had occurred outside of hospital. AKI alerts that happened $>48$ hours after admission were classified as hospital acquired. The arbitrary use of 48 hours has been applied previously in other studies and provides a means by which community-acquired AKI can be broadly categorised and assessed independently of hospital-acquired AKI. ${ }^{14}$ The admissions excluded patients under the age of 18, admissions for episodes of dialysis and pregnant patients.

These data were downloaded from Qlikview every month and presented in statistical process control charts to clearly display any statistical improvements as per the aims detailed above.

\section{Collaborative}

The QI project was based on the International Health Institute's Breakthrough Series collaborative model, ${ }^{3}$ and this model has been successfully used within the Trust previously in numerous other projects.

The SRFT AKI Collaborative Steering group was established in July 2014 in response to the recognition of AKI as a risk to patients and the rising profile of AKI on the patient safety agenda. A National Patient Safety Alert issued in 2014 mandated the introduction of a standardised computerised AKI detection algorithm.

The Collaborative steering group comprised QI facilitators, a clinical research fellow, pharmacists, practice 
Table 1 Learning sessions and dates

\begin{tabular}{ll}
\hline Learning session $\mathbf{1}$ & $\mathbf{1 7}$ August $\mathbf{2 0 1 5}$ \\
\hline Learning session 2 & 10 November 2015 \\
Learning session 3 & 10 March 2016 \\
Learning session 4 & 8 June 2016 \\
Learning session 5 & 3 October 2016 \\
\hline
\end{tabular}

\begin{tabular}{ll}
\hline Table 2 & Ward abbreviation and specialty \\
\hline Ward & Specialty \\
\hline ANU & Acute neurology unit \\
B1 & General surgical \\
B6 & Orthopaedic/trauma ward \\
B8 & Neurosurgery ward \\
EAU & Emergency assessment unit \\
H2 & Respiratory ward \\
HCU & Heart care unit \\
L2 & Gastroenterology ward \\
L5 & Care of the elderly ward \\
SHDU & Surgical high dependency unit \\
Pharmacy team & \\
\hline
\end{tabular}

educators, Information Management and Technology representatives, nursing representatives and the pre-existing AKI working group (a self-selected group of interested consultants from nephrology, acute medicine and intensive care). The steering group held a structured meeting every fortnight.

\section{Baseline data}

Baseline data were collected from November 2014 to August 2015. Pilot work, including an audit, had been undertaken in April 2014 to confirm the prevalence of AKI and the need for intervention. This showed that 212 patients were considered to have creatinine results indicative of AKI during that 1-month period. Of 177 AKI stage 1 events, 91 (51\%) did not progress, $48(27 \%)$ progressed to AKI 2 and 38 (21\%) progressed to AKI 3.

\section{DESIGN}

\section{Learning sessions}

Five learning sessions were used. Ten ward-based teams and one pharmacy team took part. Plan, do, study, act (PDSA) testing was used in action periods supported by QI facilitation and steering committee member ward visits (table 1).

The wards included (table 2) were selected for their high incidence of AKI and diversity across medical and surgical specialities to better understand the different challenges faced in diverse environments. It was anticipated that this would then lead to a more universally applicable and effective change package.

A driver diagram (shown in figure 1) was developed. The aims were to reduced overall AKI incidence by $10 \%$, reduce AKI progression by $25 \%$ and reduce the incidence of hospital acquired AKI by $50 \%$ by December 2016 . The interventions are detailed below. 


\section{AKI e-alerts}

AKI is detected through a rise in serum creatinine according to the KDIGO guidelines. ${ }^{13}$ This rise is translated into an alert if it meets the criteria within the national algorithm ${ }^{12}$ compared with a previous creatinine result or presumed baseline. The national introduction of e-alerts became mandatory in 2015 in all hospitals within the NHS. The e-alert went live in Salford Royal Hospital in July 2015.

E-alerts are a key intervention in the prompt recognition of AKI, and careful consideration was given to the effective communication of the e-alert to the clinician through EPR. This was made more robust by a phone call from Biochemistry to the ward where the blood sample had been taken for every new AKI 3. Recent work with AKI in the local community has shown that e-alerts coupled with education lead to an improved response time. ${ }^{15}$

\section{EPR documentation}

A series of changes to the EPR documents were developed including the introduction of a separate AKI assessment and an AKI pharmacist assessment document. These were to be completed when a patient develops an AKI and linked to the AKI bundle. There is good evidence of the effectiveness of bundle use from the 'Surviving Sepsis' campaign. However, literature regarding bundle completion suggested that convincing clinicians to complete a separate document is challenging. ${ }^{1617}$
Development of discharge advice for general practitioners to auto-populate on the discharge summary was also commenced. This was to be a safety net for patients whose AKI had not completely resolved on discharge to get re-referred to renal services or followed up in the community via the Primary Care CKD register, following the National Institute for Health and Care Excellence CKD guidelines. ${ }^{18}$ Potential challenges to this intervention were the need for strong communication links with primary care and the lack of evidence base for these recommendations that therefore left it open to challenge.

\section{Education}

A key component of the e-alert roll out was that it required supporting education and information. An e-learning package of learning resources and a question bank for the knowledge quiz was developed by the renal registrar, learning and development team and nursing staff within the working group. These were peer reviewed by the group prior to being uploaded to 'Moodle', the Trust's host site for online learning. Here, there was a series of educational materials aimed at the multidisciplinary team (MDT) to develop understanding of AKI, fluid balance and the role of early intervention. Based on verbal and written feedback, different iterations of the quiz were created for different job roles.

AKI education was introduced as a system change for healthcare assistant, nursing and pharmacist induction. It

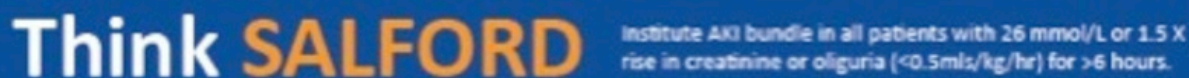

\section{Sepsis and other causes-treat}

ACE/ARB and NSAIDS suspend/review drugs

Labs (Repeat Creastinine within 24 hours) \& Leaflets (tor parsents)

Fluid assessment and response (Historv and evamination, ivitate fuid chart, measure daily

weights - if hypovolsemic give bolus IV 250 mis and reassess)

Obstruction uss should be performed within 24 hours in non-resoving AD 3 (3) rise in serum creatinine or $>354$ mmol/itre) of uniknown cause

Renal /critical care referral Non resolving AO 3, possible intrinsic renal disease requiring specialist treatment, CXO 4.5, renal transplant, severe AXI complications

\section{Dip the urine and record it}

\footnotetext{
To find the SALORD bundle to support best practice care for $A 00$ pabients on EDE:

- On EpR option panel go to preferences - Select acronym expansion - Select option export from another usera and find Nipah

- Tick acromym \#Axa, select.
}

Figure 2 Acute kidney injury (AKI) bundle poster. 
was also provided at junior doctor induction and as part of formal compulsory protected curriculum teaching to the foundation, core medical and acute care common stem medical trainees.

\section{AKI bundle document}

The Collaborative considered that the e-alert needed to trigger a tangible set of actions and interventions and provide clear advice to non-renal specialists about the referral pathway.

The renal consultants from the AKI working group developed the AKI bundle into an acronym-'SALFORD'-in the hope that this would be easily memorable for those working at SRFT (figure 2). This bundle would then tie in with the EPR documentation and formulate the response to the trigger of an AKI e-alert.

\section{AKI care app}

The AKI care app was developed and locally available in May 2015. It was designed in collaboration with the Greater Manchester, Lancashire and South Cumbria Strategic Clinical Networks to be a free, user-friendly and simple app to help guide management for AKI. It is based on the previously described detection for AKI using the national algorithm and the investigation and management of AKI using the framework of the acronym 'SALFORD' for the AKI bundle. ${ }^{19}$

\section{Pharmacy intervention}

As part of the bundle, and as an intervention in its own right, the pharmacy team planned to do a medicines reconciliation on every patient with a new AKI. An AKI medicines reconciliation pro forma was integrated into EPR. The pharmacy team and the renal physicians in the Collaborative agreed the categories that pharmacists should review. An internal aim was made that pharmacists would attempt to review medications within 24 hours of the AKI e-alert. The pharmacy team also noted which member of the parent medical team they had discussed any recommendations with.

\section{Spread phase}

Initially, the spread phase had been intended to be a threephase approach with a further 10 wards incorporated into the scheme in June 2017, followed by a full Trust roll-out. Some of the interventions in the change package, such as integration into the 'safety huddle', e-alerts and AKI education, are already being provided Trust-wide.

\section{Sustainable}

There were plans to make this sustainable from the outset. These included evaluating the successful interventions and testing these in a variety of environments, and agreeing dedicated pharmacist time to be allocated to medicines reconciliation from the outset. Education will be built into induction and teaching programmes for the entire MDT, with both face-to-face teaching and online learning. In addition, the annual 'World Kidney Day' will be used as a prompt for AKI awareness.

\section{STRATEGY}

Nominated link nurses or their representatives from the collaborative wards were invited to attend each learning session, which were facilitated by the working group. Five separate learning sessions were used to teach both the basics of AKI and QI, and then to facilitate PDSA cycles and phased implementation of practice with the 10 collaborative wards and pharmacy group. The PDSA cycles were designed to generate ward-specific small tests of change. These could be performed in individual realworld ward environments and then brought back to the group to discuss lessons, limitations and scope for adoption to other wards. The following section describes the individual topics and the phased intervention or PDSA cycles undertaken within each.

\section{AKI e-alerts}

E-alerts appear as a red text alert with AKI stage and date of AKI stage entered into EPR in the patients' demographics banner. The AKI alert is updated if the AKI stage changes but the alert does not disappear, to remind the clinician that patient remains at risk of AKI.

Some feedback was given regarding the alert remaining constantly red, and that this could be a significant cause of alert fatigue. There was also a period where the Critical Care Unit's laboratory data were not pulling through to the Qlikview, but this was recognised and rectified.

\section{Safety huddle}

Each day, nurses on each ward have a safety huddle that occurs at the changeover of shifts that include details of important safety concerns for individual patients.

PDSA 1: trialled highlighting patients with AKI and indicating AKI stage at handover.

PDSA 2: the nursing team incorporated this into the safety huddle

PDSA 3: added any outstanding aspects of the AKI bundle.

PDSA 4: feedback to the lead nurse of completed tasks from the AKI bundle during the shift.

The PDSA cycles were evaluated with qualitative feedback during phase I and the working group supported the nurses to develop a system to ensure the AKI bundle was actioned reliably. This intervention generated discussion with the responsible clinician, anecdotally improving communication around AKI. There are challenges as the e-alert does not disappear when the AKI has resolved: this requires a manual trawl by the nurse in charge of the patient of the creatinine results and AKI stage to update the handover list.

\section{AKI bundle}

Each section of the AKI bundle required individual attention and is described below (figure 2).

The conception of the bundle and the acronym 'SALFORD', development of bundle and badge-sized 'business cards' with the acronym on. Overall there was 
little interest in, or use of, the cards; however, the acronym became embedded in EPR.

There was poor engagement with completing the bundle documentation. Feedback focus groups stated that the form was not user-friendly, not intuitive and appeared to be designed for audit purposes rather than improving AKI care.

\section{Sepsis and other causes of AKI}

This part of the bundle was incorporated into a programme of education to identify and manage AKI. This is described in more detail in section 'Education: Moodle and formal teaching'.

\section{ACE-I/ARB, 'nephrotoxic' or 'volume toxic' medications}

The pharmacy team took ownership for the medicines reconciliation proforma and auditing their own performance. Alongside this, educational material and case studies were included in the medical staff education work stream.

PDSA 1: development of medicines reconciliation pro forma to audit and document recommendations, and which junior doctor this was discussed with.

Audit of this work after cycle 1 showed that $76 \%$ of patients were reviewed within 24 hours and $>90 \%$ of patients had recommendations for medication dose adjustments and $80 \%$ had a medication that was recommended to be suspended. Sixty-three per cent were taking at least one volume toxic or nephrotoxic medication. Ninety-five per cent of recommendations made by the pharmacy team were adhered to. ${ }^{20}$

PDSA 2: aimed to improve medicines reconciliation review to within 24 hours of new AKI Monday to Friday.

Unexpected benefits in this area were that the pharmacy team became their own monitors and performance regulators. They dedicated time for AKI medicines reviews and act as a human reminder for e-alerts to medical staff by documenting with whom they have discussed the medicines recommendations.

\section{Labs and leaflet}

This part of the bundle was aimed at ensuring that appropriate follow-up monitoring of creatinine (labs) was performed and that patient information was provided (leaflets). The need for a repeat creatinine was conveyed through education.

A basic patient information leaflet was designed for AKI. There were several issues with the patient information leaflet, such as determining who had the responsibility to give it to the patient and who was responsible for accompanying information such as sick day guidance or fluid guidance. The documentation of either of the above was dismal.

The reading age and language in the patient information leaflet was pitched too high for widespread comprehension. As a result, new leaflets are being developed with the help of a patient advisory group, and a short video is currently under development.

\section{Fluid balance}

Intervention: Healthcare and nursing staff formally signed over responsibility of appropriate fluid balance monitoring for patients with AKI from outgoing to incoming staff during safety huddles. This was difficult to quantify in terms of success and will need formal audit.

Specific education at induction and a healthcare-specific Moodle learning resource and quiz were developed.

\section{Obstruction}

Education sessions included reminders to doctors and nursing staff that up to $5 \%$ of AKIs are caused by obstruction and that bladder scanning or ultrasound imaging of the upper urinary tract should be considered. Ultrasound scans within 24 hours are indicated for a patient with an AKI 3 and no other obvious cause.

\section{Renal/critical care referral}

The reasons for referral were agreed by the nephrology consultants in the steering group: non-resolving AKI 3; possible intrinsic renal disease; AKI in patients with pre-existing CKD stages 4 or 5; AKI in transplant patients; severe AKI complications. Education regarding when patients should be referred to renal services were conveyed through education.

\section{Dipsticks}

Education was targeted at healthcare assistants and nurses performing and documenting fluid balance and urine dipsticks. A trial of performing urine dipstick on all patients admitted to the Medical Admissions Unit, regardless of AKI, was discontinued over concerns of an increase in inappropriate antibiotic prescriptions with minimal other changes in management.

\section{Education: Moodle and formal teaching}

A substantial programme of education was undertaken across the Trust site. This was developed in conjunction with the Trust learning and development team. Teaching events were undertaken at induction, foundation and core medical training compulsory curriculum education sessions, and for emergency village staff (doctors, nurses, advanced nurse practitioners). Online versions of these were also available, and an accompanying online quiz to test knowledge was successfully completed by over 1000 employees by December 2016 .

PDSA 1: introduction of non-mandatory online learning, completion rates showed 110 in the first month.

PDSA 2: engagement of nurse champions, some ward matrons supported the learning by withholding off-duty until staff nurses had completed it, 384 tests were completed in the following two months.

PDSA 3: AKI learning now part of mandatory induction for all staff. Foundation and core medical trainees also have additional annual face-to-face education sessions via case-based discussion. 


\section{Badges, stickers and information boxes}

Different wards decided to create different ways of highlighting or managing patients with AKI. They developed magnetic badges for the patient allocation board, the boards behind each patient bed. They also created brightly coloured filing boxes to keep together AKI-related items such as printed information like the bundle assessments, fluid balance sheets and the patient information leaflets. These were of variable success because of staff rotation, so routine use of this strategy was abandoned.

\section{AKI nurse champions}

PDSA 1: Volunteer/nominated nurse champions attended learning sessions on behalf of their wards. They underwent additional training from the QI team and the learning and development team to gain more knowledge about AKI, QI theory and support in relaying this back to their base ward.

This QI project has taken place during a period of unprecedented demand on the NHS and staffing. Recruitment and retainment are issues affecting all areas of the Trust, and, as a result, it has been especially difficult to get both regular and reliable attendance by named individuals at these organised learning sessions. Despite email reminders to both individuals and ward managers and physical walk rounds to ensure attendance, it has been increasingly difficult to maintain a turnout.

PDSA 2: The learning sessions were reduced from full day to half day or shorter sessions; this improved the numbers registered at attendance for the remainder of the sessions.

\section{Junior doctor AKI champions}

Intervention: A select group of self-declared interested foundation doctors.

Owing to fourmonthly job rotations this was significantly less effective as an intervention than anticipated. The improvement work was also not fully supported from all wards, with poor buy-in from some senior clinicians. This created a significant barrier to supporting doctors or nurses working as AKI champions within these environments.

\section{EPR AKI documentation}

Several changes to the EPR were made.

Intervention 1: AKI assessment and AKI pharmacy assessment documents.

Intervention 2: An automated insertion on to the posttake ward round for AKI assessment.

Intervention 3: Discharge documents automatically alerted the need for AKI coding.

Intervention 4: An algorithm is being developed for automated advice on phlebotomy timing after discharge, based on stage and resolution of AKI.

An audit of completion of the AKI bundle document shows that use of the AKI document within 24 hours of first AKI e-alert by medical staff is at 1.9\% (380 assessments completed for 19699 AKI episodes). This clearly indicates that the AKI document itself is not responsible for the improvements seen.

\section{RESULTS}

Over the course of the Collaborative work, there was a trend towards an increase in total episodes of AKI, in particular AKI stage 1 (figure 3). These data remained within the limits of normal variation, with an average incidence of 277 AKI episodes per month. During this same time period the median admissions per month across all inpatient wards was 2511 (minimum 2067 to maximum 2625). On Collaborative wards the median per month was 1520 (1106-1721), and for non-collaborative wards was 911 (762-1135). There was no longitudinal increase in the monthly attendances.

Trust-wide, there was a decrease in hospital-acquired AKI of $16 \%$ compared with baseline (figure 4 ). When reviewing

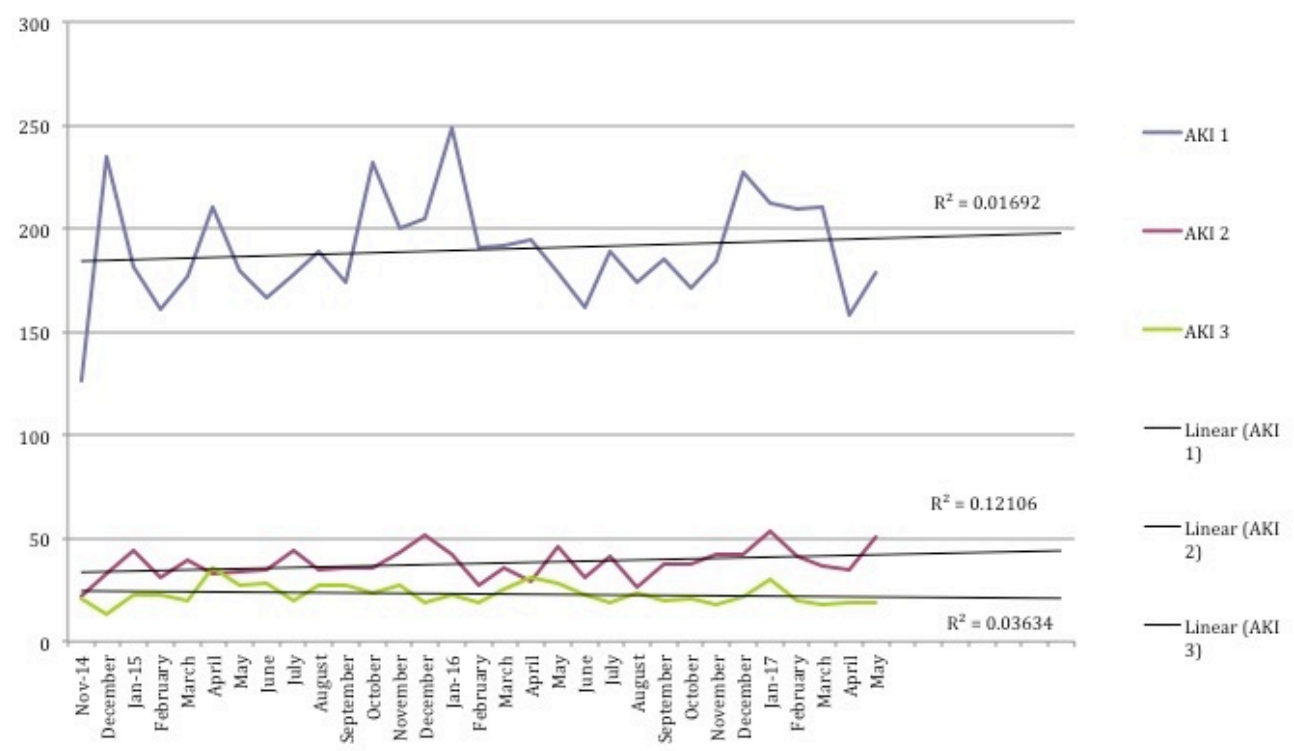

Figure 3 Number of episodes of acute kidney injury (AKI) by stage per month. 


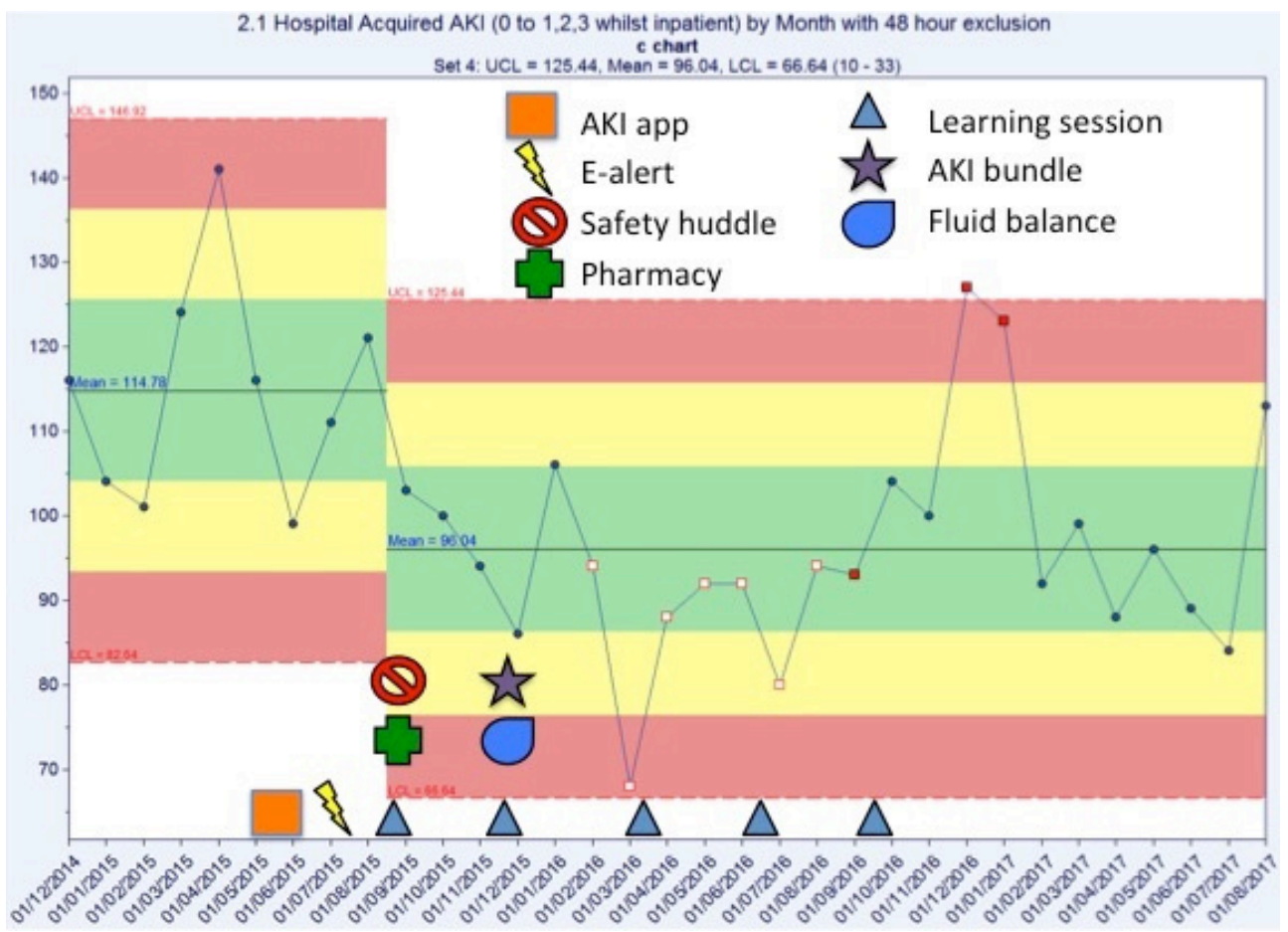

Figure 4 Number of episodes of hospital-acquired acute kidney injury (AKI) by month. There was a 16\% reduction in hospitalacquired AKI compared with baseline.

the data for the Collaborative wards separately (figure 5), the results are more pronounced: a $22 \%$ decrease in episodes of hospital-acquired AKI compared with baseline.

A review of the AKI progression (figure 6) shows monthly trends in the number of Trust-wide episodes of AKI stage 1 progressing to either AKI stage 2 or 3 , at least 48 hours after admission. There was no impact on the overall net incidence of AKI progression. However, when the data for the Collaborative wards (figure 7) were analysed independently, the number of stage AKI stage 1

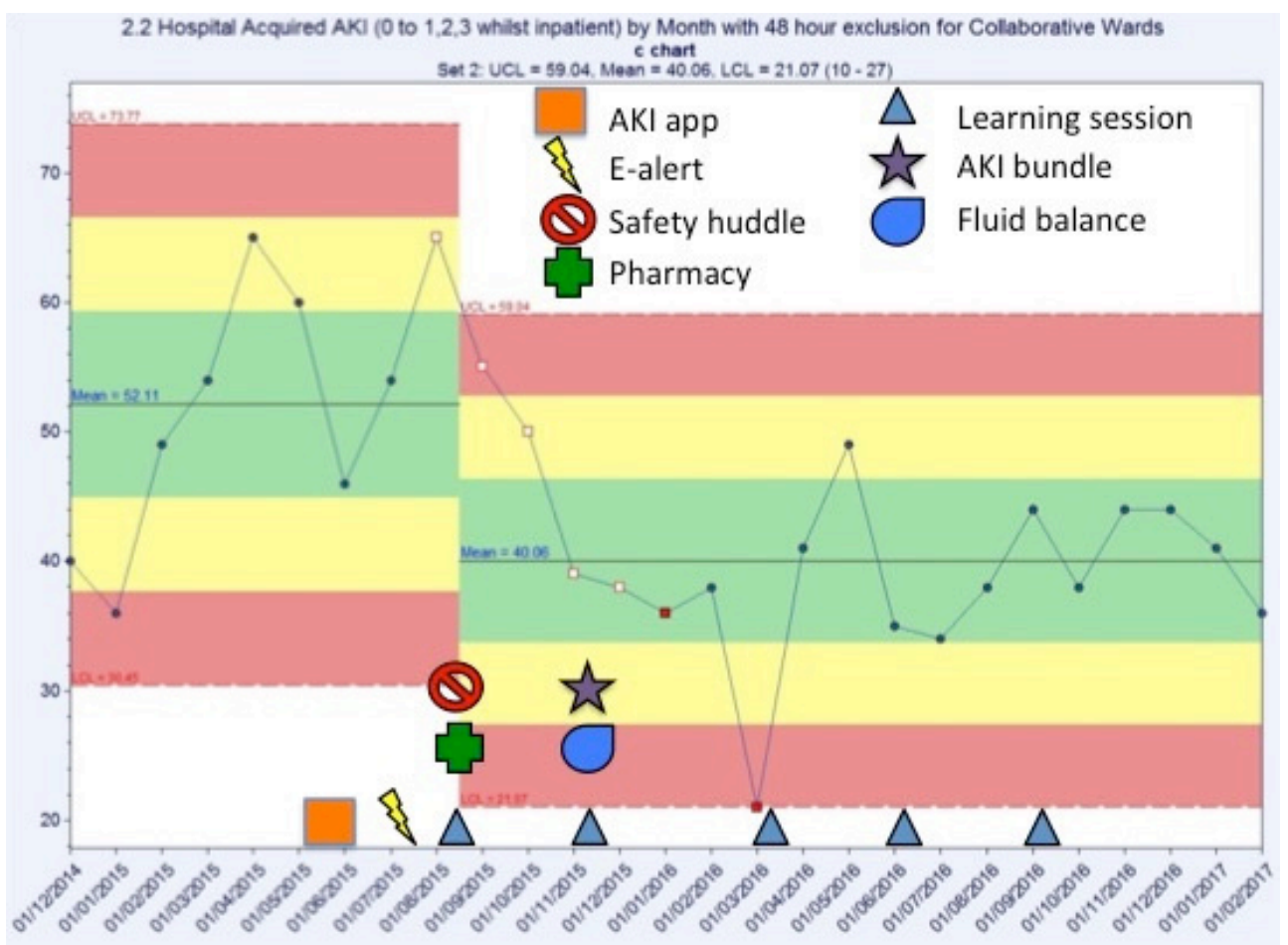

Figure 5 Number of episodes of hospital-acquired acute kidney injury (AKI) by month on the collaborative wards only. There was a $22 \%$ decrease in episodes of hospital acquired AKI compared with baseline. 


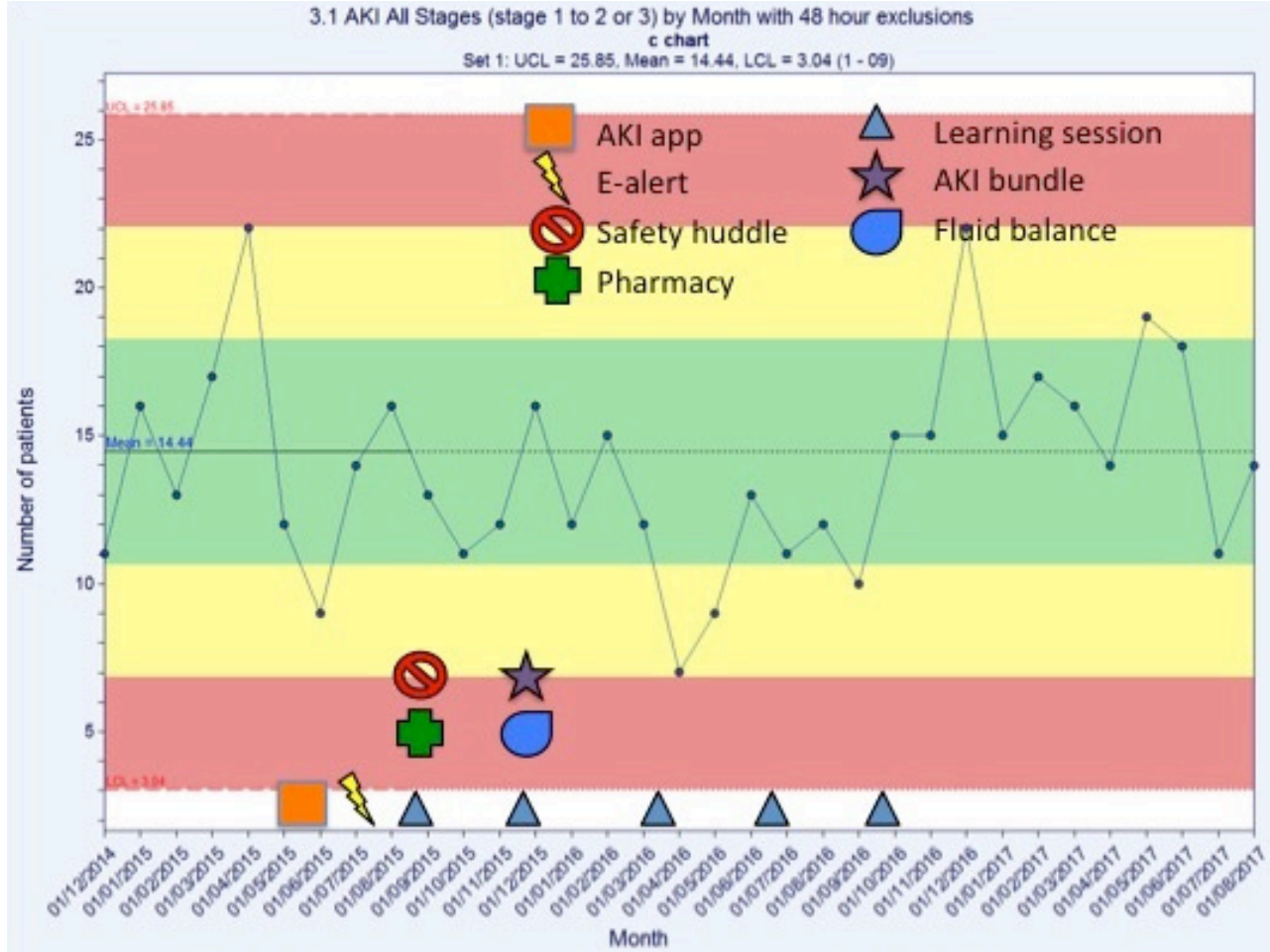

Figure 6 Number of acute kidney injury (AKI) stage 1 progressing to either AKI stage 2 or 3, 48 hours after admission, by month. This figure shows normal variation.

events that progressed to either AKI stage 2 or 3 reduced by $48 \%$ compared with baseline.

This suggests that the e-alert in isolation is ineffective at reducing AKI in this Trust. The reduction in hospital-acquired AKI and AKI progression seen on the Collaborative wards is correlated with the educational support, and the pharmacist and nurse champion work.

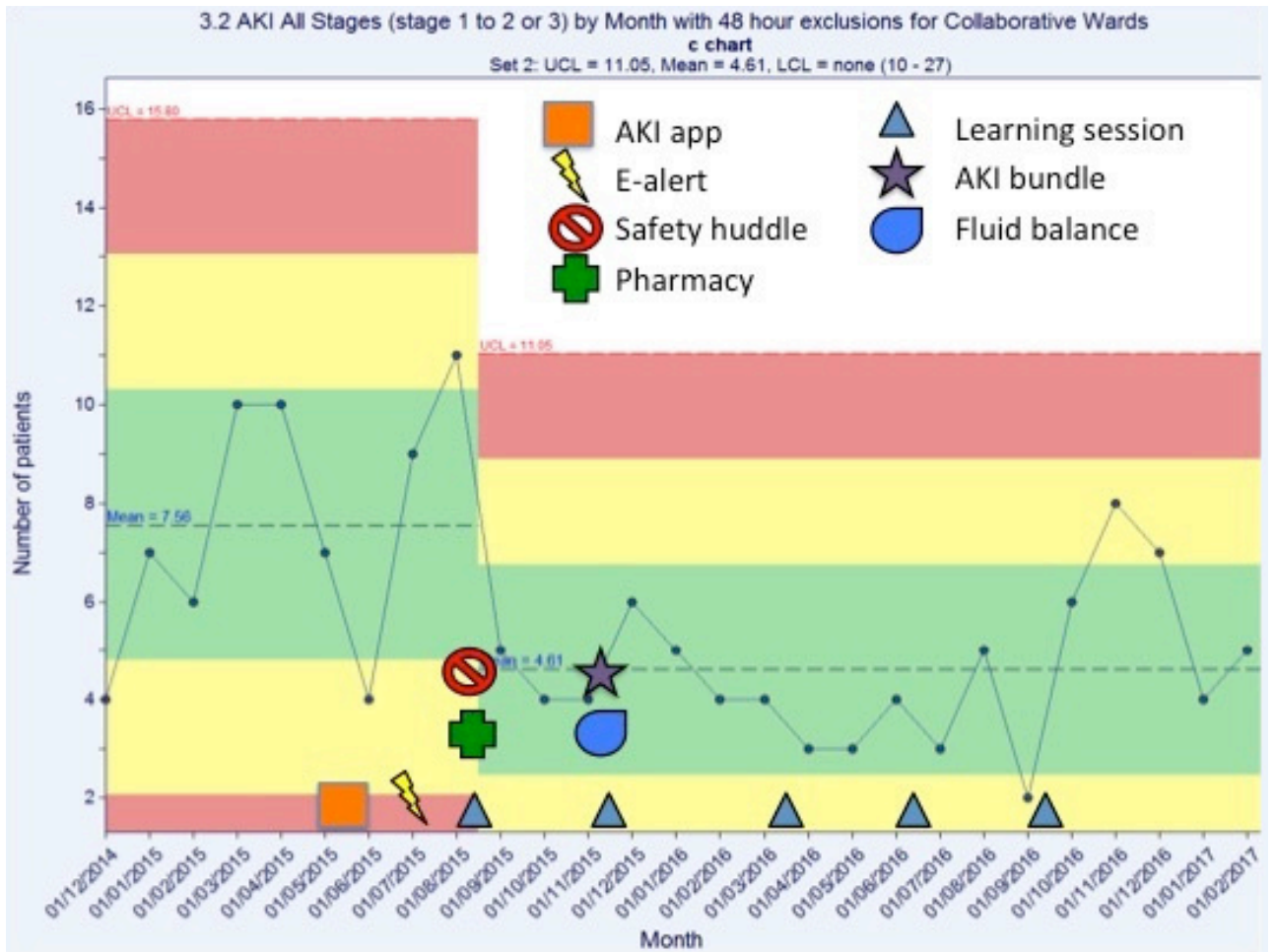

Figure 7 Number of acute kidney injury (AKI) stage 1 progressing to either AKI stage 2 or 3 on the collaborative wards only, 48 hours after admission, by month. This shows a $48 \%$ reduction in episodes on the collaborative wards compared with baseline. 
Both overall hospital length of stay and dialysis incidence show a trend towards improvement, but, owing to the wide variation, it is likely that further longitudinal data points will need to be collected in order to demonstrate whether these are statistically significant. Mortality showed no significant trend towards improvement during the study period.

\section{LESSONS AND LIMITATIONS}

There were some limitations that are both system-wide and NHS-wide. The pressures on staffing which affected the numbers of staff released to attend learning sessions, coupled with high staff turnover, impacted on the consistency and therefore knowledge base of staff present at each learning session.

We learnt that it was key to have a backbone of QI staff to organise the learning sessions, teach and facilitate QI methodology and corral input. Over the course of the project, there was reduction in frequency of meetings of the AKI working group from fortnightly to monthly and these were increasingly poorly attended. Exploration of alternative meeting times, which were no longer out of hours, was met with resistance or apathy.

There was a disappointing reception from junior doctors with regard to becoming involved in the tests of change, to bundle acceptance and completion.

Such a large and complex QI project does not lend itself easily to detailed record keeping regarding PDSA cycles, interventions and exact timings. Future projects would plan meticulous minutes taking during meetings with support of audio recording or dedicated typist and date/ time stamping of activities to enable accurate evaluation.

\section{Strengths}

There were several strengths in this project, not least the outcomes, which were statistically significant. This could not have been achieved without the stability of the QI staff support that was invaluable in arranging meetings, setting and making notes on agendas, data handling and the synthesis of the change package. The facilitation and experience of the QI staff allowed the project to run smoothly and freed up other members of the collaborative from the administrative and organisational jobs necessary for this project.

Other strengths were the engagement from the pharmacy and nursing teams as both of these were early adopters of change. The incorporation of the AKI alert into the nursing safety huddle and the pharmacy medicines reconciliation both act as a redundancy in the system for the e-alert for AKI. These are also two separate opportunities to engage with clinicians to alter management for patients and serve to make the system of recognition of AKI and its subsequent management more robust.

The whole system approach to change has resulted in the opportunity to upskill the frontline MDT not only in AKI but also the deteriorating patient and sepsis.

\section{Generalisability}

The principles of this AKI project are of a change in culture, which is low cost, easily replicated and generally applicable to all care settings. Large portions of AKI recognition tie in with other key initiatives such as early recognition of the deteriorating patient.

As many hospitals have similar nursing, pharmacy and Information Management and Technology support to SRFT, this culture change would require minimal additional expenditure. Expenditure is required for dedicated pharmacy review time, for educational training and for IT support.

Educational packages can be made universal and also be made part of the medical student curriculum as a separate module.

\section{CONCLUSION}

One of the biggest practical challenges will occur as the QI team step back from the project as this will leave a void in organisational and practical support and other members of the working group will need to step into these roles. In order to make this project robust and sustainable, there will need to be a focus in coming months on generating appropriate redundancies in the system to ensure that the statistical improvements are sustained.

Work is left to be done in the community and looking at secondary prevention as, to date, we have made no impact on incidence of community acquired AKI overall. This is likely to be because the majority of interventions are based on reactionary measures in response to e-alerts rather than risk prediction or joining forces with review of the deteriorating patient initiatives.

A number of acute hospitals have now demonstrated impactful successes in AKI reduction using traditional service improvement and QI methodologies. Almost all appear to have centred on a dedicated AKI nurse model plus e-alerting with supporting changes. This project adds value by highlighting another approach that does not require a new post with resultant rolling costs and risks. We believe that as our approach concentrated on embedding improved recognition and actions across the MDT, it has had the benefit of having increased our efficacy in acute care in our front-line teams.

Acknowledgements The authors thank members of the AKI Collaborative (full list below), all of the ward and support staff who have supported this imitative and the tests of change, and the Salford Royal NHS Foundation Trust Board for their support of this project. They also thank their patients and their relatives for sharing their AKI stories to remind them that patients are at the centre of this project.

Collaborators AKI Collaborative: Rob Nipah (Acute Medical Consultant), Liam Doyle, Chedia Hoolickin and Lewis Edwards (Quality Improvement), Dimitrios Poulikakos, Janet Hegarty and Smeeta Sinha (renal consultants), Lynne Sykes (clinical research fellow), Elizabeth Lamerton (Pharmacy), Lynn Sterling (Learning and Development), Emma Flanagan (Information Management and Technology), Paul Johnston (Electronic Patient Records), Adam Brisley and Simon Bailey (Manchester University), Paul Ferris (Intensive Care Consultant), Gareth Byrne (Clinical audit, Improving quality data), Jill Carlin (clinical director of Radiology), Denise Darby (Pathology), Debra Lee (Lead nurse), Julie Newton (lead nurse acute medicine), Gaynor White and Julie Winstanley (Emergency Admissions Unit), Susan Howard, Delphine Corgie and Zoe Ashton (NIHR CLAHRC North West. The 
National Institute for Health Research Collaboration for Leadership in Applied Health Research and Care (NIHR CLAHRC) Greater Manchester is a partnership between providers and commissioners from the NHS, industry, the third sector and the University of Manchester. We aim to improve the health of people in Greater Manchester and beyond through carrying out research and putting it into practice. (http://clahrc-gm.nihr.ac.uk). AKIcare app developers, Greater Manchester, Lancashire and South Cumbria Strategic Clinical Networks, Rachel Challiner, James Ritchie.

Contributors LS: AKI Collaborative member, developed educational material, analysed data, wrote manuscript, edited and submitted manuscript. SS: AKI working group lead, formed AKI collaborative, developed project, edited and approved final draft. JH: AKI working group and AKI collaborative member, edited and approved of final draft. EF: AKI Collaborative member, provided data support, approved final draft. LD: AKI Collaborative member, Quality Improvement lead on project, planned and delivered learning sessions, provided SPC charts, approved final draft. CH: AKI Collaborative member, planned and delivered learning sessions, approved final draft. LE joined AKI Collaborative and provided QI support, planned and delivered learning sessions, approved final draft. PF: AKI Collaborative, edited and approved final draft. EL: AKI Collaborative, lead pharmacy work stream, approved final draft. DP: AKI Collaborative member, approved final draft. DG: internal peer review, quality assurance of data, edited and approved final draft. RN: AKI lead formed AKI Collaborative, developed and lead on project, and approved final draft.

Funding The authors have not declared a specific grant for this research from any funding agency in the public, commercial or not-for-profit sectors.

Competing interests None declared.

Patient consent Not required.

Ethics approval As this was a QI study using anonymised data collection for both analysis and reporting, it is exempt from specific ethical approval.

Provenance and peer review Not commissioned; externally peer reviewed.

Open access This is an open access article distributed in accordance with the Creative Commons Attribution Non Commercial (CC BY-NC 4.0) license, which permits others to distribute, remix, adapt, build upon this work non-commercially, and license their derivative works on different terms, provided the original work is properly cited, appropriate credit is given, any changes made indicated, and the use is non-commercial. See: http://creativecommons.org/licenses/by-nc/4.0/.

\section{REFERENCES}

1. Selby NM. Electronic alerts for acute kidney injury. Curr Opin Nephrol Hypertens 2013;22:637-42.

2. Sterwart J, Findlay G, Smith N, et al. Acute kidney injury: adding insult to injury. Natl Confid Enq into Patient Outcomes Death 2009:1-22.

3. Institute for Healthcare Improvement. The breakthrough series. Innovation 2003-.
4. Public Health England. Salford health profile 2015. 2015 https://www. salford.gov.uk/media/387768/healthprofile_2015 salford.pdf.

5. Kerr M, Bedford M, Matthews B, et al. The economic impact of acute kidney injury in England. Nephrol Dial Transplant 2014;29:1362-8.

6. Finlay S, Bray B, Lewington AJ, et al. Identification of risk factors associated with acute kidney injury in patients admitted to acute medical units. Clin Med 2013;13:233-8.

7. Selby NM, Crowley L, Fluck RJ, et al. Use of electronic results reporting to diagnose and monitor $\mathrm{AKI}$ in hospitalized patients. Clin J Am Soc Nephrol2012;7:533-40.

8. Rimes-Stigare C, Frumento P, Bottai M, et al. Evolution of chronic renal impairment and long-term mortality after de novo acute kidney injury in the critically ill; a Swedish multi-centre cohort study. Crit Care2015;19:221.

9. Ponte B, Felipe C, Muriel A, et al. Long-term functional evolution after an acute kidney injury: a 10-year study. Nephrol Dial Transplant2008;23:3859-66.

10. Ebah L, Hanumapura P, Waring D, et al. A multifaceted quality improvement programme to improve acute kidney injury care and outcomes in a large teaching hospital. BMJ Qual Improv Rep 2017;6:u219176.w7476.

11. Chandrasekar T, Sharma A, Tennent L, et al. A whole system approach to improving mortality associated with acute kidney injury. QJM 2017;110:657-66.

12. NHS England. Acute Kidney Injury (AKI) Algorithm [Internet]. https:// www.england.nhs.uk/akiprogramme/aki-algorithm/ (accessed 27 Oct 17).

13. Kellum JA, Lameire N, Aspelin P, et al. Kidney disease: Improving global outcomes (KDIGO) acute kidney injury work group. KDIGO clinical practice guideline for acute kidney injury. Kidney Int 2012;2:1.

14. Singh TB, Rathore SS, Choudhury TA, et al. Hospital-acquired acute kidney injury in medical, surgical, and intensive care unit: A comparative study. Indian J Nephrol 2013;23:24-9.

15. Tollitt J, Flanagan E, McCorkindale S, et al. SO042A collaborative quality improvement project to improve management of community acquired primary care aki (capaki) using e alerts and an educational outreach programme. Nephrology Dialysis Transplantation 2017;32:iii 25-iii26.

16. Selby NM, Kolhe NV. Care bundles for acute kidney injury: do they work? Nephron 2016;134:195-9.

17. Bhagwanani A, Carpenter R, Yusuf A. Improving the management of acute kidney injury in a district general hospital: introduction of the DONUT bundle. BMJ Qual Improv Rep 2013;2:u202650.w1235.

18. NICE. Chronic kidney disease in adults: assessment and management | Guidance and guidelines. https://www.nice.org.uk/ guidance/cg182/resources (accessed 27 Oct 17).

19. Sykes L, Nipah R, Ritchie J. So044the introduction of a novel smartphone app to tackle acute kidney injury in north west england. Nephrology Dialysis Transplantation 2017;32:iii27.

20. Sykes L, Reed A, Lamerton E. Sp833evaluating pharmacist medication interventions in emergency admissions with community acquired acute kidney injury in a large teaching hospital. Nephrology Dialysis Transplantation 2017;32:iii423-iii424. 